\title{
Direct Electron Acceleration By An Intense Nonparaxial Cosh-Gaussian Laser Beam Driven Electron Plasma Wave in Plasma
}

Gunjan Purohit ( $\square$ gunjan75@gmail.com )

DAV(PG) College, Dehradun https://orcid.org/0000-0002-2694-2659

\section{Bineet Gaur}

DAV Post Graduate College Dehradun

\section{Amita Raizada}

DAV Post Graduate College Dehradun

\section{Pradeep Kothiyal}

DAV Post Graduate College Dehradun

\section{Research Article}

Keywords: Cosh-Gaussian laser beam, Relativistic-ponderomotive nonlinearity, Self-focusing, Electron plasma wave, Electron acceleration

Posted Date: July 19th, 2021

DOl: https://doi.org/10.21203/rs.3.rs-553318/v1

License: (c) (i) This work is licensed under a Creative Commons Attribution 4.0 International License. Read Full License 


\title{
Direct electron acceleration by an intense nonparaxial cosh-Gaussian laser beam driven electron plasma wave in plasma
}

\author{
Gunjan Purohit ${ }^{1}$, Bineet Gaur ${ }^{1}$, Amita Raizada ${ }^{1}$, Pradeep Kothiyal ${ }^{2}$ \\ ${ }^{1}$ Laser Plasma Computational Laboratory \\ Department of Physics, DAV (PG) College, Dehradun, Uttarakhand-248001, India \\ ${ }^{2}$ Department of Mathematics, DAV (PG) College, Dehradun, Uttarakhand-248001, India
}

Corresponding author: gunjan75@gmail.com

\begin{abstract}
Excitation of electron plasma wave by an intense short laser pulse is relevant to electron acceleration process in laser plasma interactions. In this work, the self-focusing of an intense cosh-Gaussian laser beam in collissionless plasma have been studied in the non-paraxial region with relativistic and ponderomotive nonlinearities. Further, the effect of self-focusing of the cosh-Gaussian laser beam on the excitation of electron plasma wave and on subsequent electron acceleration has been investigated. Analytical expressions for the beam width parameter/intensity of cosh-Gaussian laser beam and the electron plasma wave have been established and solved numerically. The energy of the accelerated electrons has also been obtained. The strong self-focusing of the cosh-Gaussian laser beam in plasmas stimulates a large amplitude electron plasma wave, which further accelerates the electrons. The wellestablished laser and plasma parameters have been used in numerical computation. The results have been compared with paraxial ray approximation, Gaussian profile of laser beam and only with the relativistic nonlinearity. Numerical results suggest that the focusing of the coshGaussian laser beam, the amplitude of electron plasma wave, and energy gain by electrons increases in non-paraxial region, when relativistic and ponderomotive nonlinearities are simultaneously operative. In addition, it has also been observed that the electron plasma wave is driven more efficiently by a cosh-Gaussian laser beam that accelerates plasma electrons to higher energies.
\end{abstract}

Key Words: Cosh-Gaussian laser beam, Relativistic-ponderomotive nonlinearity, Selffocusing, Electron plasma wave, Electron acceleration 


\section{Introduction}

The development of short-pulse high intensity lasers has led to research in laser-plasma interactions (Mourou et al. 2006; Norreys et al. 2009). The propagation of intense laser pulses into plasma is the subject of active research that is relevant to many prospective applications such as the acceleration of charged particles, fast ignition in inertial confinement fusion, and new radiation sources (Joshi 2017; Tanaka et al. 2000; Jaroszynski et al. 2006). Laser beams must be propagated over a several Rayleigh lengths in the plasma without divergence or loss of the energy for the realization of these applications. When an ultra-short intense laser beam propagates through the plasma, the beam itself becomes focused and produces ultra-high laser intensity over a large distance, which is used for the excitation of large amplitude electron plasma wave. These laser-driven plasma waves are used for ultra-high energies in the electron acceleration process (Modena et al. 1995; Yadav et al. 2018). Therefore, self-focusing of an intense laser beam in plasma is the most important nonlinear phenomena in laser plasma interaction.

Self-focusing is related to the distortion of the laser beam wavefront, which arises due to the modification of the dielectric constant/refractive index of the plasma during the propagation of an intense laser beam into plasma (Chekalin and Kandidov 2013; Sun et al. 1987). The modification in the dielectric constant/refractive index of the plasma depends on the nonlinearities of the plasma. At high laser intensity, the dielectric constant/refractive index of the plasma is mainly modified by ponderomotive nonlinearity and relativistic nonlinearity. Ponderomotive nonlinearity arises due to the electron density perturbations by the ponderomotive force of the beam. This nonlinearity is important in the self-focusing of the laser beam due to the expulsion of electrons from the focal spot. The ponderomotive force increases the dielectric constant/refractive index of the plasma, which leads to strong selffocusing of the laser beam. On the other hand, relativistic nonlinearity is set up due to intense electric field of the laser beam. The quiver motion of electrons increases due to high electric field of the laser beam, which further enhances the relativistic mass of electrons and the dielectric constant/refractive index of the plasma.

Self-focusing of laser beam in the plasma and its effect on the excitation of electron plasma wave and on the electron acceleration process have been studied extensively in the past (Tajima and Dawson 1979; Clayton et al. 1993, Everett et al. 1994; Nakajima et al. 1995; Singh and Gupta 2003; Liu and Tripathi 2005; Kumar et al. 2006; Esarey et al. 2009; Leemans and Esarey 2009; Priyanka et al. 2013; Gaur et al. 2016; Xia et al. 2017; Vranic et al. 2018; Yadav 
et al. 2020; Raynaud et al. 2020). The extent of self-focusing of an intense laser beams in plasmas and the amplitude of electron plasma wave depends on the spatial profile of laser beams and the nonlinearities associated with plasma. Most of these studies have been confined to the Gaussian distribution of the laser beams under paraxial-ray approximation, where the effect of ponderomotive nonlinearity and relativistic nonlinearity have been taken separately. Nevertheless, various profiles of laser beams such as super Gaussian beams (Gill et al. 2015), elliptic Gaussian beams (Gaur et al. 2018), Hermite-Gaussian beams (Wadhwa and Singh 2020), hollow Gaussian beams (Purohit et al. 2016), Hermite-cosh-Gaussian laser beams (Belafhal and Ibnchaikh 2000), and q-Gaussian beams (Yadav et al. 2020) have been used in a few studies of self-focusing and plasma wave excitation. Such beams having different types of irradiance across their wavefront, which show different features in plasma. Recently, flattop decentred cosh-Gaussian laser beams have attracted much attention because of their higher efficient power and attractive applications (Konar et al. 2007; Aggarwal et al. 2014; Habibi and Ghamari 2015). One of the most important specialty of cosh-Gaussian laser beam is that it becomes focus earlier than Gaussian laser beam. In addition, it has also been observed that when an intense laser beam propagates through the plasma, both relativistic and ponderomotive nonlinearities act simultaneously, which significantly enhance the self-focusing of the laser beam in plasma and the amplitude of electron plasma wave. Furthermore, paraxial-ray theory does not adequately describe the self-focusing of laser beams in plasma at high intensity. The paraxial-ray approximation has failed to describe the variation of radial profile of the beam from the initial to ring position. On the other hand, non-paraxial ray approximation (Sodha and Faisal 2008; Gill et al. 2010) accurately describes the propagation of an intense laser beam in plasma, where the eikonal and nonlinear dielectric constant extends up to the fourth power of the distance from the axis of beam. Therefore, non- paraxial approximation is pertinent for cosh Gaussian laser beam along with relativistic and ponderomotive nonlinearities.

The excitation of electron plasma wave by an intense laser beam is a useful approach to electron acceleration. In the present study, we have investigated the self-focusing of an intense non-paraxial cosh-Gaussian laser beam in collisionless plasma in the presence of relativistic and ponderomotive nonlinearities. The effect of the self-focused cosh-Gaussian laser beam on the excitation of electron plasma wave as well as the electron acceleration has also been studied. The numerical results shows that the focusing of cosh-Gaussian laser beam is strong in non-paraxial region. Due to strong focusing of cosh-Gaussian laser beam in plasma, a dexterous high amplitude electron plasma wave is excited which enhances the energy of accelerated electrons. The structure of the paper is as follows: In section 2, the appropriate 
expressions for the nonlinear effective dielectric constant of the plasma as well as for the selffocusing of a high-intensity cosh-Gaussian laser beam through collisionless plasma has been derived under the nonparaxial ray approximation, when both relativistic and ponderomotive nonlinearities are operative. The modified equations for the excitation of electron plasma wave and for the electron acceleration process has been acquired in section 3. A brief discussion of the numerical results based on derived equations are presented in section 4 . Finally, the main conclusions are summarized in the section 5 .

\section{Basic formulation}

An intense cosh- Gaussian laser beam is considered to be propagates in $z$-direction through collisionless unmagnetized plasma. The laser electric field at $z=0$ under non-paraxial approximation is given by

$$
E(r, 0)=\frac{E_{00}^{2}}{2 f} e^{\left(\frac{b^{2}}{4}\right)}\left[\exp \left\{-\left(\frac{r}{r_{0}}+\frac{b}{2}\right)^{2}\right\}+\exp \left\{-\left(\frac{r}{r_{0}}-\frac{b}{2}\right)^{2}\right\}\right] \times\left(1+\frac{r^{2}}{r_{0}^{2}} a_{20}(z)+\frac{r^{4}}{r_{0}^{4}} a_{40}(z)\right)
$$

where $r$ is the radial coordinate of the cylindrical coordinate system, $r_{0}$ is the initial beam width of the beam, $E_{00}$ is the amplitude of the electric field at the central position of $r=z=0, b$ is the decentred parameter of the beam, $a_{20}(z)$ and $a_{40}(z)$ are the coefficient of $r^{2}$ and $r^{4}$ and are indicative for the departure of the beam from the Gaussian nature.

\subsection{Effective dielectric constant of the plasma}

The dielectric constant of plasma at high laser intensity can be written as

$$
\varepsilon_{0}=1-\frac{\omega_{p 0}^{2}}{\omega_{0}^{2}} \frac{1}{\gamma}
$$

where $\omega_{p 0}^{2}=\frac{4 \pi n_{0} e^{2}}{m_{0}}$ is the plasma frequency, $\omega_{0}$ is the frequency of the laser beam, $n_{0}$ is the density of plasma electrons in the absence of laser beam, $e$ and $m_{0}$ are the charge and rest mass of electron respectively. The relativistic factor $(\gamma)$ can be written as

$$
\gamma=\left(1+\frac{e^{2}}{c^{2} m_{0}^{2} \omega_{0}^{2}} E \cdot E^{*}\right)^{1 / 2}
$$

In the present study, the electron density and the dielectric constant of plasma are being modified by cumulative effect of relativistic and ponderomotive nonlinearities. These nonlinearities modify the dielectric constant of plasma by relativistic increment in the electron 
mass and by the ponderomotive expulsion of electrons from the beam path. An intense laser beam exerts the relativistic-ponderomotive force on the electrons, which expels the electrons away from the region of higher electric field and modifies the background electron density. The relativistic-ponderomotive force is given by (Sun et al. 1987; Brandi et al. 1993)

$$
F_{R P}=-m_{0} c^{2} \nabla(\gamma-1)
$$

The modified density of plasma electrons $(n)$ due to relativistic-ponderomotive force is given as (Brandi et al. 1993)

$$
\frac{n_{e}}{n_{0}}=1+\frac{c^{2}}{\omega_{p 0}^{2}}\left(\nabla^{2} \gamma-\frac{(\nabla \gamma)^{2}}{\gamma}\right)=1+\frac{c^{2}}{\omega_{p 0}^{2}}\left[\frac{a}{2 f^{2}}\left(\frac{A}{C^{1 / 2}}-\frac{a}{2 f^{2}} \frac{B}{C^{3 / 2}}\right)-\frac{a^{2}}{4 f^{4}} \frac{B}{C^{3 / 2}}\right]
$$

where,

$a\left(=\alpha E_{00}^{2}\right)$ is the initial intensity of the beam

$$
\begin{aligned}
& A=4 \frac{P_{1}}{f^{2} r_{0}^{2}}+16 \frac{P_{2}}{f^{4} r_{0}^{4}} r^{2}+36 \frac{P_{3}}{f^{6} r_{0}^{6}} r^{4} \\
& B=4 \frac{P_{1}^{2}}{f^{4} r_{0}^{4}} r^{2}+16 \frac{P_{1} P_{2}}{f^{6} r_{0}^{6}} r^{4}
\end{aligned}
$$

and

$$
C=1+\frac{c^{2}}{f^{2}}\left\{1+\frac{P_{1}}{f^{2} r_{0}^{2}} r^{2}+\frac{P_{2}}{f^{4} r_{0}^{4}} r^{4}\right\}
$$

with

$$
\begin{aligned}
& P_{1}=b^{2}+a_{20}-2 \\
& P_{2}=a_{40}+a_{20} b^{2}-2 a_{20}+b^{4} / 3-2 b^{2}+2 \\
& P_{3}=b^{6}-14 b^{4}+48 b^{2}+8 a_{20} b^{4}-48 b^{2}+24 a_{20} b^{2}-48 a_{40}+16
\end{aligned}
$$

The effective intensity dependent dielectric constant of plasma can be written as

$$
\varepsilon(r, z)=\varepsilon_{0}+\phi\left(E \cdot E^{*}\right)
$$

where

$$
\phi\left(E \cdot E^{*}\right)=\frac{\omega_{p 0}^{2}}{\omega_{0}^{2}}\left(1-\frac{n_{e}}{n_{0} \gamma}\right)
$$

In the non-paraxial region, the dielectric function can be expressed as

$$
\varepsilon(r, z)=\varepsilon_{0}(z)-\frac{r^{2}}{r_{0}^{2}} \varepsilon_{2}(z)-\frac{r^{4}}{r_{0}^{4}} \varepsilon_{4}(z)
$$

where $\varepsilon_{0}(z), \varepsilon_{2}(z)$ and $\varepsilon_{4}(z)$ are the expansion coefficients. 


\subsection{Self-focusing of non-paraxial cosh-Gaussian laser beam in plasma}

The electric field $E$ of the laser beam in the plasma satisfies the following wave equation: $\nabla^{2} E-\nabla(\nabla \cdot E)+\frac{\omega_{0}^{2}}{c^{2}} \varepsilon E=0$

where $\varepsilon$ the intensity dependent dielectric constant of the plasma, and $E$ is the electric field of the beam. Consider the variation of the electric field can be expressed as

$$
E(r, z)=A(r, z)\left[\exp i\left(\omega_{0} t-k_{0} z\right)\right]
$$

where $A$ is the amplitude of the laser field, and $k_{0}(z)=\frac{\omega_{0}}{c} \sqrt{\varepsilon_{0}(z)}$ is the wave vector of the beam.

By replacing Eq. (10) in Eq. (9) and neglecting the term $\frac{\partial^{2} A}{\partial z^{2}}$ (assuming slow variation of $A$ versus $z$ ), one obtains

$$
\frac{\partial^{2} A}{\partial r^{2}}+\frac{1}{r} \frac{\partial A}{\partial r}-2 i k_{0} \frac{\partial A}{\partial z}-i A \frac{\partial k_{0}}{\partial z}+\frac{\omega_{0}^{2}}{c^{2}}\left[\varepsilon(r, z)-\varepsilon_{0}\right] A=0
$$

Further, the complex amplitude $A(r, z)$ can be expressed as [33]

$$
A(r, z)=A_{0}(r, z) \exp \left[-i k_{0}(z) S_{0}(r, z)\right]
$$

where $A_{0}$ is the beam irradiance, and $S_{0}$ is the eikonal i. e. the real function of space, which describes converging/diverging behaviour of the beam in the plasma.

Substituting Eqs. (8) \& (12) in Eq. (11) and separating the real and imaginary parts, following equations are obtained:

$$
2 \frac{\partial S_{0}}{\partial z}+\frac{2 S_{0}}{k_{0}} \frac{d k_{0}}{d z}+\left(\frac{\partial S_{0}}{\partial r}\right)^{2}=\frac{1}{k_{0}^{2} A_{0}}\left(\frac{\partial^{2} A_{0}}{\partial r^{2}}+\frac{1}{r} \frac{\partial A_{0}}{\partial r}\right)-\frac{r^{2}}{r_{0}^{2}} \frac{\varepsilon_{2}(z)}{\varepsilon_{0}(z)}-\frac{r^{4}}{r_{0}^{4}} \frac{\varepsilon_{4}(z)}{\varepsilon_{0}(z)}
$$

and

$$
\frac{\partial A_{0}^{2}}{\partial z}+A_{0}^{2}\left(\frac{\partial^{2} S_{0}}{\partial r^{2}}+\frac{1}{r} \frac{\partial S_{0}}{\partial r}\right)+\frac{\partial A_{0}^{2}}{\partial r} \frac{\partial S_{0}}{\partial r}+\frac{A_{0}^{2}}{k_{0}} \frac{d k_{0}}{d z}=0
$$

The solution of Eq. (14) under non-paraxial approximation can be written as (Sodha and Faisal 2008; Gill et al. 2010)

$$
A_{0}^{2}=\frac{E_{00}^{2}}{4 f^{2}} \exp \left(\frac{b^{2}}{2}\right)\left(1+\frac{a_{20} r^{2}}{r_{0}^{2} f^{2}}+\frac{a_{40} r^{4}}{r_{0}^{4} f^{4}}\right)\left[\exp \left\{-\left(\frac{r}{r_{0} f}+\frac{b}{2}\right)^{2}\right\}+\exp \left\{-\left(\frac{r}{r_{0} f}-\frac{b}{2}\right)^{2}\right\}\right]^{2}
$$

where $f$ is the beam width parameter of the cosh-Gaussian laser beam. 
The eikonal $S_{0}(r, z)$ can be expressed as

$$
S_{0}(r, z)=S_{10}(z)+\frac{r^{2}}{r_{0}^{2}} S_{20}(z)+\frac{r^{4}}{r_{0}^{4}} S_{40}(z)
$$

where $S_{10}(z)$ is the axial phase shift, $S_{20}(z)$ and $S_{40}(z)$ are indicative of the spherical curvature of the wavefront and its departure from the spherical nature, respectively.

Substituting Eqs. (15) and (16) into Eq. (14), one can obtain

$$
\begin{aligned}
& S_{20}=\frac{r_{0}^{2}}{2 f} \frac{d f}{d z} \\
& \frac{\partial a_{20}}{\partial \xi}=-16 S_{40}^{\prime} f^{2}
\end{aligned}
$$

and

$$
\frac{\partial a_{40}}{\partial \xi}=-8 S_{40}^{\prime} f^{2}\left(b^{2}+3 a_{20}-2\right)
$$

where $\xi\left(=\frac{c z}{\omega_{0} r_{0}^{2}}\right)$ is the dimensionless propagation distance of laser beam and $S_{40}^{\prime}=S_{40} \frac{\omega_{0}}{c}$ By eliminating $S_{40}^{\prime}$ from Eqs. (18) and (19) and integrating the resulting equation with the initial conditions $a_{40}=0$ and $a_{20}=0$ at $\xi=0$, one obtains the relation between $a_{40}$ and $a_{20}$ is as:

$$
a_{40}=\frac{\left(4 b^{2} a_{20}+3 a_{20}^{2}-4 a_{20}\right)}{4}
$$

Replacing the value of $a_{40}$ from Eq. (20) to Eq. (15), we obtain the following equation for the intensity of cosh-Gaussian laser beam:

$$
\begin{aligned}
& A_{0}^{2}=E \cdot E^{*}=\frac{E_{00}^{2}}{4 f^{2}} \exp \left(\frac{b^{2}}{2}\right)\left(1+\frac{a_{20} r^{2}}{r_{0}^{2} f^{2}}+\frac{\left(4 b^{2} a_{20}+3 a_{20}^{2}-4 a_{20}\right) r^{4}}{4 r_{0}^{4} f^{4}}\right) \\
& \times\left[\exp \left\{-\left(\frac{r}{r_{0} f}+\frac{b}{2}\right)^{2}\right\}+\exp \left\{-\left(\frac{r}{r_{0} f}-\frac{b}{2}\right)^{2}\right\}\right]^{2}
\end{aligned}
$$

By replacing Eqs. (15) \& (16) in Eq. (13) and equating the coefficients of $r^{2}$ and $r^{4}$ on both sides of the resulting equation, following differential equations are obtained for $f$ and $S_{40}^{\prime}$

$$
\frac{d^{2} f}{d \xi^{2}}=\frac{\chi_{1}}{\varepsilon_{0} f^{3}}+\frac{\rho_{0}^{2} a}{2 \varepsilon_{0}}\left[\frac{P_{1}}{\gamma^{3} f^{3}}+\frac{c^{2}}{\omega_{p 0}^{2} f}\left\{\frac{16 P_{2}}{r_{0}^{2} \gamma^{2} f^{4}}-\frac{6 a P_{1}^{2}}{r_{0}^{2} \gamma^{4} f^{6}}-\frac{2 a P_{1}}{\gamma^{4} f^{4}}\right\}\right]
$$

and 
$\frac{d S_{40}^{\prime}}{d \xi}=\frac{\chi_{2}}{\varepsilon_{0} f^{6}}-\frac{4 S_{40}^{\prime}}{f} \frac{d f}{d \xi}-\frac{S_{40}^{\prime}}{2 \varepsilon_{0}} \frac{d \varepsilon_{0}}{d \xi}-\frac{\rho_{0}^{2}}{2 \varepsilon_{0}} \varepsilon_{4}$

where

$$
\begin{aligned}
& \chi_{1}=8 a_{40}+2 a_{20} b^{2}-3 a_{20}^{2}-4 a_{20}-\frac{b^{4}}{3}-4 b^{2}+4 \\
& \chi_{2}=4 a_{20}^{3}+4 a_{20}^{2}+4 a_{40} b^{2}-2 a_{20}^{2} b^{2}-8 a_{40}-14 a_{20} a_{40}-2 a_{20} \frac{b^{4}}{3}+17 \frac{b^{4}}{6}+\frac{b^{6}}{12}
\end{aligned}
$$

and $\rho_{0}=\frac{r_{0} \omega_{0}}{c}$ is the original beam width of the beam.

Self-focusing of a cosh-Gaussian laser beam is given by nonlinear differential equation (22), where the first term on right hand side represents the diffractional divergence of the beam, and the second term describes the convergence of the beam, which arises due to combined effect of ponderomotive and relativistic nonlinearities. The focusing/defocusing of laser beam in the plasma depends on the relative magnitude of these two terms. The solution for selffocusing of cosh Gaussian laser beam can be obtained by simultaneous solution of Eqs. (17) (20) and Eq. (22)-(23) with initial conditions $f=1, d f / d \xi=0, S_{40}^{\prime}=0$ and $a_{20}=0$ at $\xi=0$.

Substituting Eq. (21) in Eq. (6), expanding $\varepsilon$ as a series of power $r / r_{0}$, and comparing the result with Eq. (8), one can obtain the expression for the parameters $\varepsilon_{0}, \varepsilon_{2}$, and $\varepsilon_{4}$ as:

$$
\begin{aligned}
& \varepsilon_{0}=\varepsilon_{0}(r, z)_{r=0}=1-\frac{\omega_{p 0}^{2}}{\omega_{0}^{2} \gamma}+\frac{2 c^{2} a P_{1}}{\omega_{0}^{2} r_{0}^{2} \gamma^{2} f^{4}} \\
& \varepsilon_{2}=-\left(\frac{\partial \varepsilon_{0}(r, z)}{\partial r^{2}}\right)_{r=0}=-\frac{\omega_{p 0}^{2}}{\omega_{0}^{2}}\left[\frac{P_{1} a}{2 r_{0}^{2} \gamma^{3} f^{4}}+\frac{c^{2} a}{2 \omega_{p 0}^{2} f^{2}}\left\{\frac{16 P_{2}}{r_{0}^{4} \gamma^{2} f^{4}}-\frac{6 a P_{1}^{2}}{r_{0}^{4} \gamma^{4} f^{6}}-\frac{2 a P_{1}}{r_{0}^{2} \gamma^{4} f^{4}}\right\}\right]
\end{aligned}
$$

and

$$
\varepsilon_{4}=-\frac{\partial}{\partial r^{2}}\left(\frac{\partial \varepsilon_{0}(r, z)}{\partial r^{2}}\right)_{r=0}=-\frac{\omega_{p 0}^{2}}{\omega_{0}^{2}}\left[\frac{P_{2} a}{r_{0}^{2} \gamma^{3} f^{6}}-\frac{3 a P_{1}^{2}}{4 r_{0}^{4} \gamma^{5} f^{8}}+\frac{c^{2} a}{2 \omega_{p 0}^{2} f^{2}}\left\{\frac{3 P_{3}}{r_{0}^{6} \gamma^{2} f^{6}}-\frac{72 a P_{1} P_{2}}{r_{0}^{4} \gamma^{2} f^{4}}+\frac{8 a^{2} P_{1}^{2}}{r_{0}^{4} \gamma^{6} f^{8}}\right\}\right]
$$

\section{Excitation of electron plasma wave}

The cosh-Gaussian laser beam becomes self-focused in the plasma when the initial power of laser beam is greater than the critical power. The intensity of laser beam becomes very high at 
the focused positions. This is due to the modification in electron density of plasma by ponderomotive force and the relativistic effects. Such intense laser beams further excite the electron plasma wave. The amplitude of electron plasma wave depends on the background electron density of plasma. Thus, the electron plasma wave coupled to high intensity laser beam via modified background electron density. In order to analyse the effect of this coupling on the excitation of electron plasma wave, we start with the following set of equations:

(a) Equation of continuity:

$$
\frac{\partial N_{e}}{\partial t}+\nabla \cdot\left(N_{e} V\right)=0
$$

(b) Equation of motion:

$$
m\left[\frac{\partial v_{e}}{\partial t}+(V \cdot \nabla) V\right]=-e E-\frac{e}{c} V \times B-2 \Gamma_{e} m V-\frac{\gamma_{i} k_{B} T_{0}}{N_{e}} \nabla N_{e}
$$

and

(c) Poisson's equation

$$
\nabla \cdot E=-4 \pi e N_{e}
$$

where $N_{e}\left(=N_{0 e}+n_{e 0}\right)$ is the total electron density, $V\left(=V_{0}+v\right)$ is the sum of the electron velocities in the electromagnetic and self-consistent field, $v$ is the drift velocity of the electron, $E$ and $B$ are the electric and magnetic fields, $\Gamma_{e}$ is the Landau damping factor for electron plasma wave, $\gamma_{I}(=3)$ is the ratio of specific heat and other symbols have their usual meaning. In order to obtain large amplitude electron plasma wave, $\Gamma_{e}$ can be neglected.

Using Eqs. (27-29), one obtains the general equation governing the electron density variation in the electron plasma wave (neglecting the contribution of the ions) as:

$$
\frac{\partial^{2} n_{e 0}}{\partial t^{2}}+2 \Gamma_{e} \frac{\partial n_{e 0}}{\partial t}-v_{t h}^{2} \nabla^{2} n_{e 0}+\frac{\omega_{p 0}^{2}}{\gamma} \frac{n_{e}}{n_{0}} n_{e 0}=0
$$

where $v_{t h}^{2}=\left(\frac{k_{B} T_{0}}{m_{0}}\right)^{1 / 2}$ is the electron thermal velocity. The solution of Eq. (30) can be written as

$$
n_{e 0}=n_{e 00}(r, z) \exp \left\{-i\left[\omega_{e} t-k_{e} z-S_{e}(r, z)\right]\right\}
$$

where $n_{e 00}$ is the slowly varying real function of $r$ and $z, S_{e}$ is the eikonal of the plasma wave, $\omega_{e}$ and $k_{e}$ are the frequency and the propagation vector of the electron plasma wave related by the following dispersion relation 
$\omega_{e}^{2}=\omega_{p 0}^{2}\left(\frac{n_{e}}{n_{0}}\right)+3 k_{e}^{2} v_{t h}^{2}$

By replacing Eq. (31) into Eq. (30) and separating the real and imaginary part, one

gets

$2 \frac{\partial S_{e}}{\partial z}+\left(\frac{\partial S_{e}}{\partial r}\right)^{2}=\frac{1}{k_{e}^{2} n_{e 00}}\left(\frac{\partial^{2} n_{e 00}}{\partial r^{2}}+\frac{1}{r} \frac{\partial n_{e 00}}{\partial r}\right)+\frac{\omega_{p 0}^{2}}{3 k_{e}^{2} v_{t h}^{2}}\left(1-\frac{n_{e}}{n_{0} \gamma}\right)$

and

$\frac{\partial n_{e 00}^{2}}{\partial z}+\frac{\partial S_{e}}{\partial r} \frac{\partial n_{e 00}^{2}}{\partial r}+n_{e 00}^{2}\left(\frac{\partial^{2} S_{e}}{\partial r^{2}}+\frac{1}{r} \frac{\partial S_{e}}{\partial r}\right)+\frac{2 \Gamma_{e} \omega_{e}}{3 k_{e} v_{t h}^{2}} n_{e 00}^{2}=0$

The solution ofEqs. (33) and (34) under non-paraxial approximation can be written as (Sodha and Faisal 2008; Gill et al. 2010)

$n_{e 00}^{2}=\frac{N_{e 00}^{2}}{4 f_{e}^{2}} \exp \left(\frac{b^{2}}{2}\right)\left[\exp \left\{-\left(\frac{r}{f_{e} r_{e 0}}+\frac{b}{2}\right)^{2}\right\}+\exp \left\{-\left(\frac{r}{f_{e} r_{e 0}}-\frac{b}{2}\right)^{2}\right]^{2}\left(1+\frac{a_{2 e} r^{2}}{f_{e}^{2} r_{e 0}^{2}}+\frac{a_{4 e} r^{4}}{f_{e}^{4} r_{e 0}^{4}}\right) e^{-2 k_{i} z}\right.$

where $r_{e 0}$ is the initial beam width of the plasma wave and $N_{10}$ is the initial density associated with the electron plasma wave at $r=0$,

and

$S_{e}=\frac{S_{2 e} r^{2}}{r_{e 0}^{2}}+\frac{S_{4 e} r^{4}}{r_{e 0}^{4}}$

where $S_{2 e}=\frac{r_{e 0}^{2}}{2 f_{e}} \frac{d f_{e}}{d z}, k_{i}=\frac{2 \Gamma_{e} \omega_{0}}{k_{e} v_{t h}^{2}}, a_{2 e}$ and $a_{4 e}$ are the contributions from the $r^{2}$ and $r^{4}$ coefficients and are the functions of $z, S_{2 e}$ and $S_{4 e}$ are eikonal contribution from $r^{2}$ and $r^{4}$ coefficients in non-paraxial region and are functions of $(r, z)$.

Now using Eqs. (35) and (36) in Eq. (33) and equating the coefficients of $r^{2}$ and $r^{4}$ on both sides, we obtain the following equations:

$\frac{d^{2} f_{e}}{d \xi^{2}}=\frac{\chi_{1}^{\prime}}{\varepsilon_{0} f_{e}^{3}}\left(\frac{k_{0}}{k_{e}}\right)^{2} \frac{r_{0}^{4}}{r_{e 0}^{4}}+f_{e}\left(\frac{k_{0}}{k_{e}}\right)^{2}\left(\frac{c^{2}}{v_{t h}^{2}}\right) \frac{\rho^{2} a}{2 \varepsilon_{0}}\left[\frac{P_{1}}{f^{4} \gamma^{3}}+\frac{c^{2}}{f^{2} \omega_{p 0}^{2}}\left\{\frac{16 P_{2}}{r_{0}^{2} f^{4} \gamma^{2}}-\frac{6 a P_{1}^{2}}{r_{0}^{2} f^{6} \gamma^{4}}-\frac{2 a P_{1}}{f^{4} \gamma^{4}}\right\}\right]$

and

$\frac{d S_{4 e}}{d \xi}=\frac{\chi_{2}^{\prime}}{\varepsilon_{0} f_{e}^{6}}\left(\frac{k_{0}}{k_{e}}\right)^{2} \frac{r_{0}^{2}}{r_{e 0}^{2}}-\left(\frac{k_{0}}{k_{e}}\right)^{2} \frac{\rho_{0}^{2}}{2 \varepsilon_{0}} \varepsilon_{4}-\frac{4}{f_{e}} \frac{d f_{e}}{d z} S_{4 e}$ 
Similarly, by substituting (35) and (36) in Eq. (34) and equating the coefficients of $r^{2}$ and $r^{4}$ on both sides of the equation, we obtain the following equations:

$\frac{\partial a_{2 e}}{\partial \xi}=-16 S_{4 e}^{\prime}(z) f_{e}^{2}$

and

$\frac{\partial a_{4 e}}{\partial \xi}=-8 S_{4 e}^{\prime}(z) f_{e}^{2}\left(b^{2}+3 a_{2 e}-2\right)$

where

$$
\begin{aligned}
& \chi_{1}^{\prime}=8 a_{4 e}+2 a_{2 e} b^{2}-3 a_{2 e}^{2}-4 a_{2 e}-\frac{b^{4}}{3}-4 b^{2}+4 \\
& \chi_{2}^{\prime}=4 a_{2 e}^{3}+4 a_{2 e}^{2}+4 a_{4 e} b^{2}-2 a_{2 e}^{2} b^{2}-8 a_{4 e}-14 a_{2 e} a_{4 e}-2 a_{2 e} \frac{b^{4}}{3}+17 \frac{b^{4}}{6}+\frac{b^{6}}{12}
\end{aligned}
$$

and

$$
S_{4 e}^{\prime}(z)=S_{4 e} \frac{\omega_{0}}{c}
$$

By eliminating $S_{4 e}^{\prime}$ from Eqs. (39) \& (40) and integrating the resulting equation with the initial conditions $a_{4 e}=0$ and $a_{2 e}=0$ at $\xi=0$, one obtains

$$
a_{4 e}=\frac{\left(4 b^{2} a_{2 e}+3 a_{2 e}^{2}-4 a_{2 e}\right)}{4}
$$

The initial conditions for $f_{e}$ are: $f_{\mathrm{e}}=1$ and $d f_{\mathrm{e}} / d z=0$, at $z=0$, and $S_{2 \mathrm{e}}=S_{4 e}=0$ at $z=0$.

\subsection{Electron acceleration}

The excited electron plasma wave by an intense cosh-Gaussian laser beam transfers its energy to electrons and accelerates them. The energy gain by the electron is given by

$\gamma=\left(1-\frac{v^{2}}{c^{2}}\right)^{-1 / 2}$

The equation of motion for an electron can be written as

$$
\frac{d(m v)}{d t}=i k_{e} m_{0} c^{2} \phi_{e}
$$

Differentiating Eq. (42) and using Eq. (43), we get

$$
\frac{d \gamma}{d t}=-i v k_{e} \phi_{e}
$$

where $\phi_{e}=\frac{e \phi}{m_{0} c^{2}}$ is the dimensionless electrostatic potential of electron plasma wave.

Using Poisson's equation, one can obtains the expression for $\phi_{e}$ 
$\phi_{e}=\frac{i \omega_{p 0}^{2}}{c^{2} k_{e}^{2} f_{e}} \exp \left(-k_{i} z\right) \sin (k z)$

Eq. (44) has been solved numerically by using Eq. (37) to obtain energy gain by electrons.

\section{Numerical results and discussion}

To investigate the self-focusing behaviour of cosh-Gaussian laser beam in unmagnetized plasma under non-paraxial region and its effect on the plasma wave excitation and on electron acceleration, we have solved above coupled equations by computer simulations. The following set of laser and plasma parameters have been used in numerical computation:

$\omega_{0}=1.778 \times 10^{15}, r_{0}=15 \mu \mathrm{m}, \omega_{p 0}=0.04 \omega_{0}, 0.06 \omega_{0}$, and $0.08 \omega_{0}, b=0,0.4,0.6$, and 0.8 , and $a$ $\left(\alpha E_{00}{ }^{2}\right)=1,1.4$, and 2 . The initial boundary conditions are:

$f=1, d f / d \xi=0, S_{40}^{\prime}=0$ and $a_{20}=0$ at $\xi=0$

$f_{e}=1, d f_{e} / d \xi=0, S_{4 \mathrm{e}}^{\prime}=0$ and $a_{2 \mathrm{e}}=0$ at $\xi=0$

Equations (21) and (22) describe the intensity profile and beamwidth (focusing/defocusing) of cosh-Gaussian laser beam in the plasma, when both the relativistic and ponderomotive nonlinearities are operative. It is clear from Eq. (21) that the intensity profile of cosh-Gaussian laser beam in nonparaxial region depends on the beamwidth parameter $(f)$ and the coefficients of $r^{2}$ (i. e. $\left.a_{20}\right)$ and $r^{4}$ (i. e. $a_{40}$ ). The numerical computation of the Eqs. $(18,19,22$ and 23) has been performed to understand the self-focusing behaviour of coshGaussian laser beam in the plasma. In order to obtain the variation in the intensity of coshGaussian laser beam with the normalized distance of propagation ( $\xi$ ), Eq. (21) have been solved numerically along with the numerical computation of Eqs. (18, 19, 22 and 23) respectively, and the results are illustrated in Figures (1-4).

Figure (1) depicts the variation in normalized intensity of cosh-Gaussian laser beam with normalized distance of propagation, when relativistic and ponderomotive nonlinearities are operative in paraxial and non-paraxial regions. The laser beam becomes self-focused when propagates through the plasma. It is obvious that the intensity of cosh-Gaussian laser beam enhances in non-paraxial region in comparison to the paraxial region due to the influence of the off-axial coefficients. This is due to the fact that the focusing of laser beam becomes strong and fast in nonparaxial region in comparison to the paraxial region. Figure (2) presents the variation of normalized intensity of cosh-Gaussian laser beam in the plasma along the propagation distance in nonparaxial region, when only relativistic and relativisticponderomotive nonlinearities are operative. It is observed that the self-focusing of cosh- 
Gaussian laser beam becomes significantly enhanced by the inclusion of ponderomotive nonlinearity. Due to the strong focusing of the cosh-Gaussian laser beam by relativisticponderomotive nonlinearity, the intensity of the beam is remarkably increases as compared to only relativistic nonlinearity. Figure (3) shows the effect of decentred parameter $(b)$ on normalized intensity of cosh-Gaussian laser beam in plasma. The laser beam exhibits Gaussian profile at $b=0$. It is observed that with an increase in the value of $b$, the extent of self-focusing increases. Consequently, the intensity of cosh-Gaussian laser beam in the plasma increases at higher values of $b$. From $b=0$ to $b=0.8$, the maximum intensity of the laser beam gets enhanced by a factor of about 2.5. Thus, the fast-converging behaviour of cosh-Gaussian laser beam leads us to pick the cosh-Gaussian profile of laser beam. Figure (4) represents the variation of normalized intensity of cosh-Gaussian laser beam in plasma with the normalized propagation distance in non-paraxial region for different values of intensity parameter $(a)$. The extent of self-focusing of the beam is also increases at higher values of $a$. It is obvious from the Figure that with the increase in the value of $a$, the intensity of laser beam is increases.

The amplitude of the electron plasma wave (i. e. electron density perturbation) excited by cosh-Gaussian laser beam is given by Equation (35). Equation (37) describes the coupling of the electron plasma wave with the cosh-Gaussian laser beam in presence of the relativistic and ponderomotive nonlinearities. In order to obtain the amplitude of the electron plasma wave with the normalized propagation distance, Eq. (35) have been solved numerically with the help of Eqs. (37-40). The amplitude of the electron plasma wave depends on $f, f_{e}$, and the off-axial coefficients ( $a_{20}$ and $a_{40}$ ) of $r^{2}$ and $r^{4}$ in the nonparaxial region. Figure (5) illustrates normalized intensity of electron plasma wave with the normalized propagation distance in paraxial and non-paraxial regions at fixed values of $b, a$, and $\omega_{p 0}$, when both the relativistic and ponderomotive nonlinearities are operatives. Due to strong nonlinear coupling between coshGaussian laser beam and the electron plasma wave in non-paraxial region, the intensity of electron plasma wave is increases. Figure (6) displays the electron plasma wave intensity with normalized distance of propagation in non-paraxial region, when only relativistic nonlinearity and both relativistic and ponderomotive nonlinearities are taken into consideration. The combined effect of ponderomotive and relativistic nonlinearity significantly enhances the intensity of electron plasma wave in comparison to only relativistic nonlinearity. It is obvious from Figure 6 that the intensity of electron plasma wave gets enhanced by a factor of about 8 in non-paraxial region, when both nonlinearities are operative. The variation of the normalized electron plasma wave intensity with the normalized propagation distance in non-paraxial region at different values of $b$ is shown in Figure (7), when the relativistic and ponderomotive 
nonlinearities are simultaneously functional. Due to the contribution of $f, f_{e}$, and off-axial components, the intensity of electron plasma wave increases at higher values of $b$. Moreover, the intensity/amplitude of electron plasma wave is maximum at those points where the laser beam is focused. A large amplitude electron plasma wave becomes excited at focused positions of the laser beam. Figure 8 represents the variation in the normalized electron plasma wave intensity with the normalized distance of propagation in non-paraxial region at different values of intensity parameter $(a)$, when both nonlinearities are present. Again, the intensity of electron plasma wave increases with increase the value of $a$. This is due to the fact that the electron density perturbation increases at higher values of $a$, which further enhances the amplitude of electron plasma wave.

The high amplitude electron plasma wave transmits its energy in electron acceleration process. In order to see the effect of high amplitude electron plasma wave on the electron acceleration process, Eq. (44) have been solved numerically and the results are depicted in Figs. (9-12). The results show that the energy gain by electrons depends on the beam width parameters of the laser beam and electron plasma wave, off-axial components, nonlinearities associated with the laser beam, amplitude of electron plasma wave, decentered parameter $(b)$, and intensity parameter $(a)$. It is clear from Fig. (9) that the energy gain is significantly enhances in non-paraxial region, when the relativistic and ponderomotive nonlinearities are perused together. This is because the amplitude of the laser beam and electron plasma wave is be higher in the non-paraxial region. Figure (10) shows that the amplitude of the electron plasma wave gets enhances by a factor of about 9 by inclusion of ponderomotive nonlinearity with relativistic nonlinearity. Figure (11) represents the variation of energy gain with the normalized propagation distance in the non-paraxial region for different values of $b$ in the presence of relativistic and ponderomotive nonlinearities. It is found that with the increase in the value of $b$, energy gain by electrons is remarkably increases. This is due to the fact that cosh-Gaussian laser beam excites larger amplitude electron plasma wave at higher values of $b$ (Fig. 7). It is obvious from Fig. 11 that at the optimized value of $b=0.8$, the maximum electron energy gain is achieved. Figure (12) shows the effect of intensity parameter $(a)$ on the energy gain by electrons in the nonparaxial region, when both the relativistic and the ponderomotive nonlinearities are operative. Due to the increase in the amplitude of electron plasma wave at higher values of $a$ (Fig. 8), the energy gain also increases along with the increase in the value of $a$. 


\section{Conclusions}

In summary, we have studied the self-focusing of cosh-Gaussian laser beam in a collisionless unmagnetized plasma under non-paraxial ray approximation along with the combined effect of relativistic and ponderomotive nonlinearities. Further, the effect of the self-focusing of coshGaussian laser beam on the excitation of electron plasma wave and the electron acceleration have been examined. The results have been compared with the paraxial-ray approximation and with the relativistic nonlinearity. It has been found that the extent of self-focusing of coshGaussian laser beam becomes stronger and faster in the non-paraxial region and in the relativistic-ponderomotive regime. The amplitude/intensity of the electron plasma wave and the energy gain by the electrons are also increases in the non-paraxial region and in the relativistic-ponderomotive regime. Moreover, self-focusing/intensity of cosh-Gaussian laser beam in plasma becomes enhances at higher values of $b$ and $a$. Consequently, the amplitude/intensity and the energy gain by electrons also increases with increase in the values of $b$ and $a$ respectively. Due to earlier and strong self-focusing of cosh-Gaussian laser beam at higher values of $b$, the intensity of laser beam, the amplitude/intensity of electron plasma wave and the energy gain by electrons becomes remarkably enhanced. Therefore, on the basis of these results it is suggested that the Gaussian laser beam might be replaced by the coshGaussian laser beam, which can be further used for electron plasma wave excitation and more effectively for electron acceleration.

Conflicts of interest: The authors declare that they have no conflict of interest.

Data availability: The data can be obtained on demand. 


\section{References}

Agarwal, M., Vij, S., Kant, N.: Propagation of cosh Gaussian laser beam in plasma with density ripple in relativistic - ponderomotive regime. Optik 125(18), 5081-5084(2014)

Belafhal, A., Ibnchaikh, M.: Propagation properties of Hermite-cosh-Gaussian laser beams. Opt. Commun. 186(4-6), 269-276(2000)

Brandi, H. S., Manus, C., Mainfray, G. Relativistic and ponderomotive self-focusing of a laser beam in a radially inhomogeneous plasma. I. Paraxial approximation. Phys. Fluids B 51993(10), 3539-3550(1993)

Brandi, H. S., Manus, C., Mainfray, G., Lehner, T.: Relativistic self-focusing of ultra-intense laser pulses in inhomogeneous underdense plasmas. Phys. Rev. E 47(5), 3780-3783(1993)

Chekalin, S. V., Kandidov, V. P.: From self-focusing light beams to femtosecond laser pulse filamentation. Physics -Uspekhi 56(2), 123-140(2013)

Clayton, C. E., Marsh, K. A., Dyson, A., Everett, M., Lal, A., Leemans, W. P., Williams, R., Joshi, C.: Ultrahigh-gradient acceleration of injected electrons by laser-excited relativistic electron plasma waves. Phys. Rev. Lett. 70(1), 37-40(1993)

Esarey, E., Schroeder, C. B., Leemans, W. P.: Physics of laser-driven plasma-based electron accelerators. Rev. Mod. Phys. 81(3), 1229-1285(2009)

Everett, M., Lal, A., Gordon, D., Clayton, C. E., Marsh, K. A., Joshi, C. Trapped electron acceleration by a laser-driven relativistic plasma wave. Nature 368(6471), 527-529(1994)

Gaur, B., Rawat, P., Purohit, G., Effect of self-focused cosh Gaussian laser beam on the excitation of electron plasma wave and particle acceleration. Laser Part. Beams 34(4), 621630(2016)

Gaur, B., Rawat, P., Purohit, G.: Mitigation of stimulated Raman backscattering by elliptical laser beam in collisionless plasma. Optik 157(3), 99-112(2018)

Gill, T. S., Kaur, R., Mahajan, R.: Propagation of high-power electromagnetic beam in relativistic magnetoplasma: Higher order paraxial ray theory. Phys. Plasmas 172010(9), 093101(2010)

Gill, T. S., Kaur, R., Mahajan, R.: Self-focusing of super-Gaussian laser beam in magnetized plasma under relativistic and ponderomotive regime. Optik 126(18), 1683-1690(2015)

Habibi, M., Ghamari, F.: Improved Focusing of a cosh-Gaussian Laser Beam in Quantum Plasma: Higher Order Paraxial Theory. IEEE Trans. Plasma Sci. 43(7), 2160-2165(2015) 
Jaroszynski, D. A., Bingham, R., Brunetti, E., Ersfeld, B., Gallacher, J., Geer B. van der, Issac, R., Jamison, S. P., Jones, D., Loos, M. de, Lyachev, A., Pavlov, V., Reitsma, A, Saveliev, Y., Vieux, G., Wiggins, S. M.: Radiation sources based on laser-plasma interactions. Phil. Trans. R. Soc. A.364(1840), 689-710(2006).

Joshi, C.: Laser-Driven Plasma Accelerators Operating in the Self-Guided, Blowout Regime. IEEE Trans. Plasma Sci. 45(12), 3134-3146(2017).

Konar, S., Mishra, M., Jana, S.: Nonlinear evolution of cosh-Gaussian laser beams and generation of flat top spatial solitons in cubic quintic nonlinear media. Phys Lett. A 362(5-6), 505-510(2007)

Kumar, A., Gupta, M. K., Sharma, R. P.: Effect of ultra-intense laser pulse on the propagation of electron plasma wave in relativistic and ponderomotive regime and particle acceleration. Laser Part. Beams 24(3), 403-409(2006).

Leemans, W., Esarey, E. Laser-driven plasma-wave electron accelerators. Phys. Today 62(3), 44-50(2009).

Liu, C. S., Tripathi, V. K.: Ponderomotive effect on electron acceleration by plasma wave and betatron resonance in short pulse laser. Phys. Plasmas 12(4), 043103(2005).

Modena, A., Najmudin, Z., Dangor, A. E., Clayton, C. E., Marsh, K. A., Joshi, C., Malka, V., Darrow, C. B., Danson, C., Neely, D., Walsh, F. N.: Electron acceleration from the breaking of relativistic plasma waves. Nature 377(6550), 606-608(1995)

Mourou, G. A., Tajima, T., Bulanov, S. V.: Optics in the relativistic regime. Rev. Mod. Phys. 78(2), 309-371(2006)

Nakajima, K., Fisher, D., Kawakubo, T., Nakanishi, H., Ogata, A., Kato, Y., Kitagawa, Y., Kodama, R., Mima, K., Shiraga, H., Suzuki, K., Yamakawa, K., Zhang, T., Sakawa, Y., Shoji, T., Nishida, Y., Yugami, N., Downer, M., Tajima, T.: Observation of ultrahigh gradient electron acceleration by a self-modulated intense short laser pulse. Phys. Rev. Lett. 74(22), 44284431(1995)

Norreys, P. A., Beg, F. N., Sentoku, Y., Silva, L. O., Smith, R. A., Trines, R. M. G. M.: Intense laser-plasma interactions: New frontiers in high energy density physics. Phys. Plasmas 16(4), 041002(2009)

Priyanka, Chauhan, P., Purohit, G.,: Relativistic ponderomotive effect on the propagation of rippled laser beam and the excitation of electron plasma wave in collisionless plasma. Opt. Commun. 311(1), 317-324(2013)

Purohit, G., Rawat, P., Gauniyal, R.: Second harmonic generation by self-focusing of intense hollow Gaussian laser beam in collisionless plasma. Phys. Plasmas 23(1), 013103(2016) 
Raynaud, M., Héron, A., Adam, J. C.: Excitation of surface plasma waves and fast electron generation in relativistic laser-plasma interaction. Sci. Rep. 10(1), 13450(2020)

Singh, K. P. Gupta, V. L.: Electron acceleration by a plasma wave in a sheared magnetic field. Phys. Plasmas 10(5), 1493-1499(2003)

Sodha, M. S., Faisal, M.: Propagation of high-power electromagnetic beams in overdense plasmas: Higher order paraxial theory. Phys. Plasmas 15(3), 033102(2008)

Sun, G. Z., Ott, E., Lee, Y. C., Guzdar, P.: Self-focusing of short intense pulses in plasmas. Phys. Fluids 30(2), 526-532(1987)

Tajima, T., Dawson, J. M.: Laser Electron Accelerator. Phys. Rev. Lett. 43(4), 267-269(1979).

Tanaka, K. A. et al.: Studies of ultra-intense laser plasma interactions for fast ignition. Phys. Plasmas 7(5), 2014-2022(2000)

Vranic, M., Fonseca, R. A., Silva, L. O., Extremely intense laser-based electron acceleration in a plasma channel. Plasma Phys. Control. Fusion 60(3), 034002(2018)

Wadhwa, J., Singh, A.: Enhanced second harmonic generation of Hermite-Gaussian laser beam in plasma having density transition. Laser Phys. 30(4), 046001(2020)

Xia, X., Liang, Q., Gao, R., Wang, L.: Relativistic ponderomotive force acceleration of electrons in weak inhomogenous underdense plasma. Optik 136(1), 20-26(2017)

Yadav, M., Sharma, S. C., Gupta, D. N.: Electron acceleration by a relativistic electron plasma wave in inverse-free-electron laser mechanism. IEEE Trans. Plasma Sci. 46(7), 25212527(2018)

Yadav, M., Gupta, D. N., Sharma, S. C.: Electron plasma wave excitation by a q-Gaussian laser beam and subsequent electron acceleration. Phys. Plasmas 27(9), 093106(2020) 


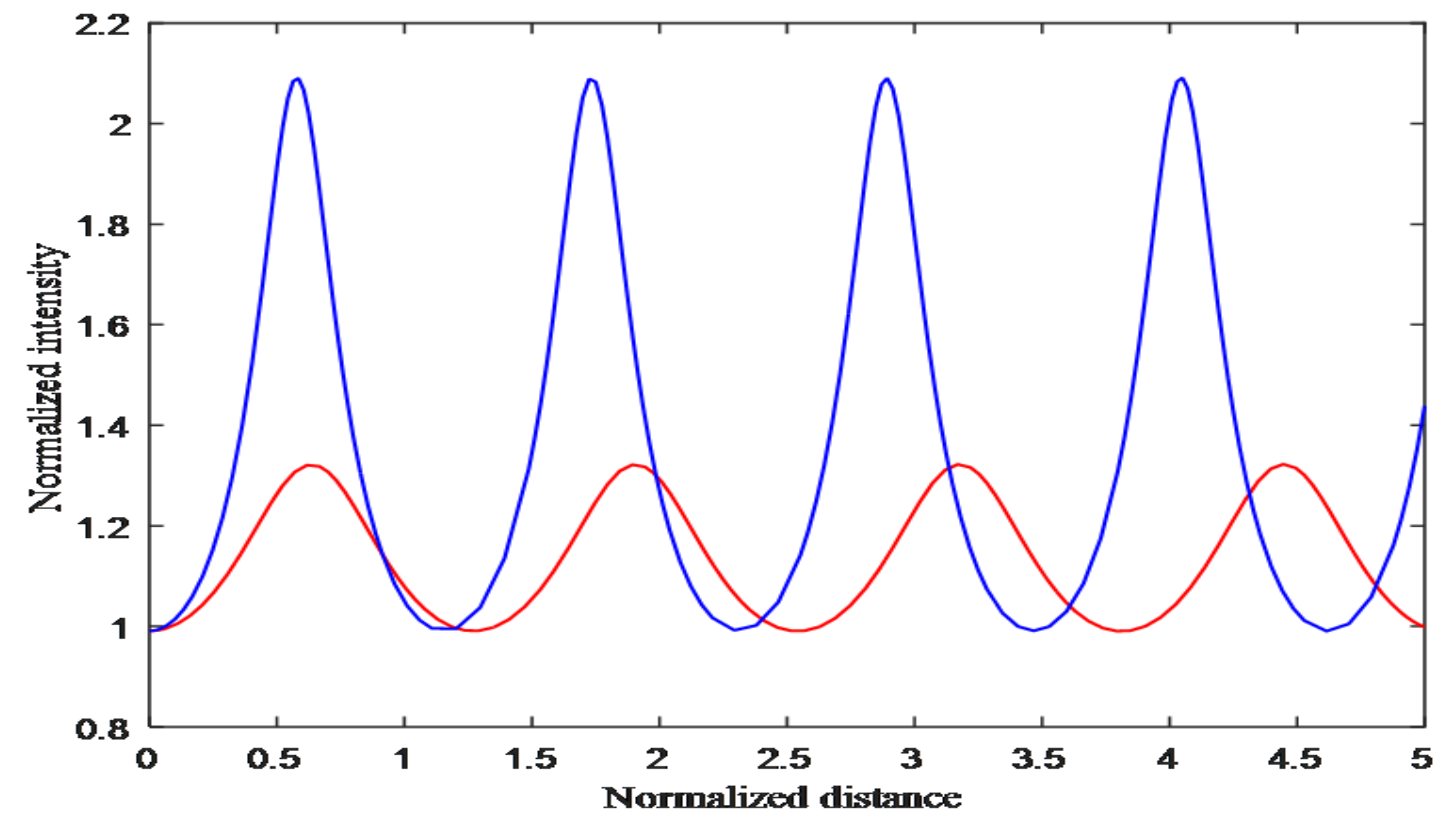

Figure 1. Variation of the normalized intensity of cosh-Gaussian laser beam in the plasma with normalized propagation distance $(\xi)$. Keeping $a=1.4, b=0.6$, and $\omega_{p 0}=0.06 \omega_{0}$, when relativistic and ponderomotive nonlinearities are operative. Red curve for paraxial region and blue curve for non-paraxial region.

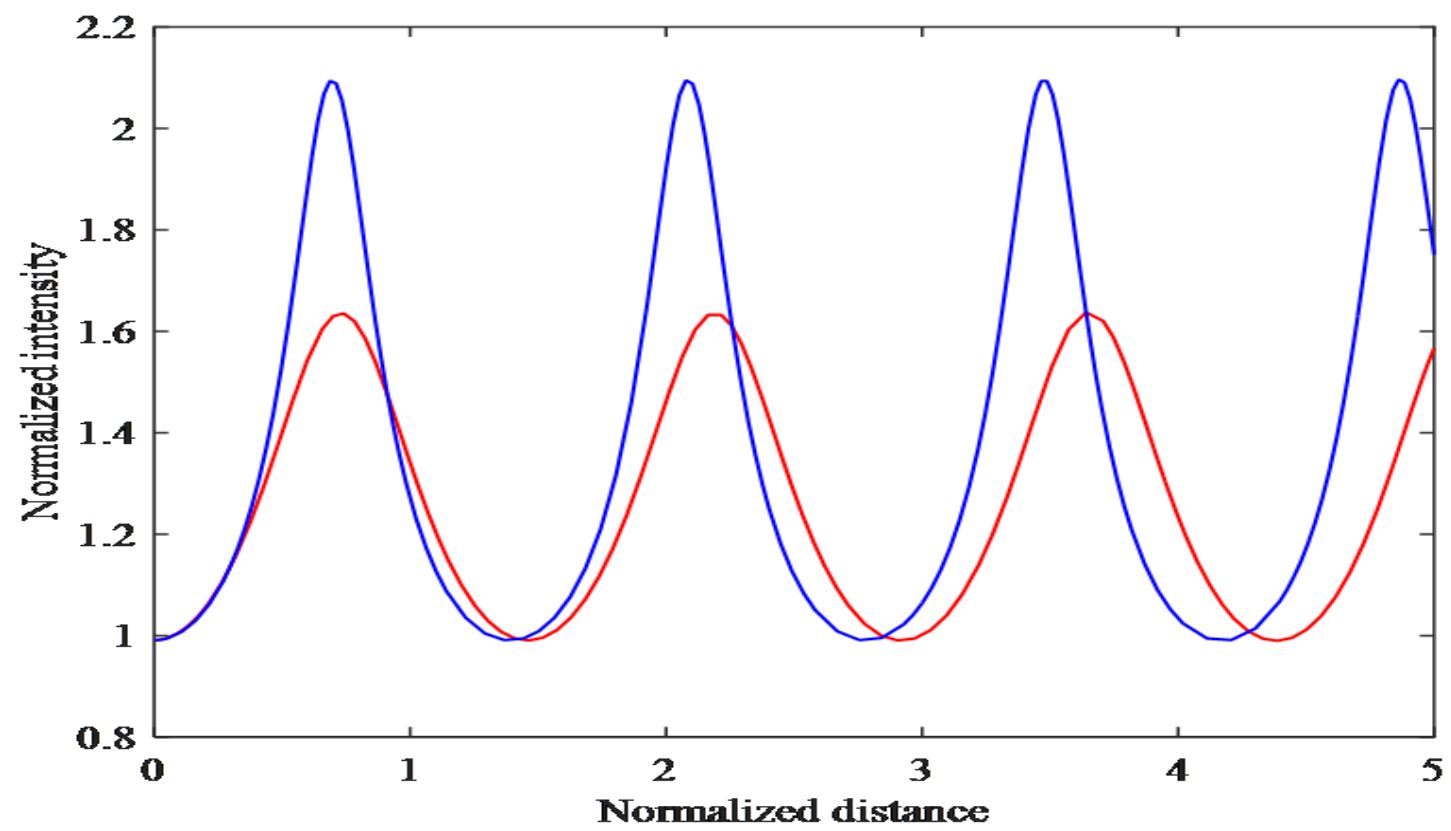

Figure 2. Variation of the normalized intensity of cosh-Gaussian laser beam in the plasma with normalized propagation distance $(\xi)$. Keeping $a=1.4, b=0.6$, and $\omega_{p 0}=0.06 \omega_{0}$, when relativistic and ponderomotive nonlinearities are operative. Red curve for only relativistic and blue curve for relativistic-ponderomotive nonlinearities. 


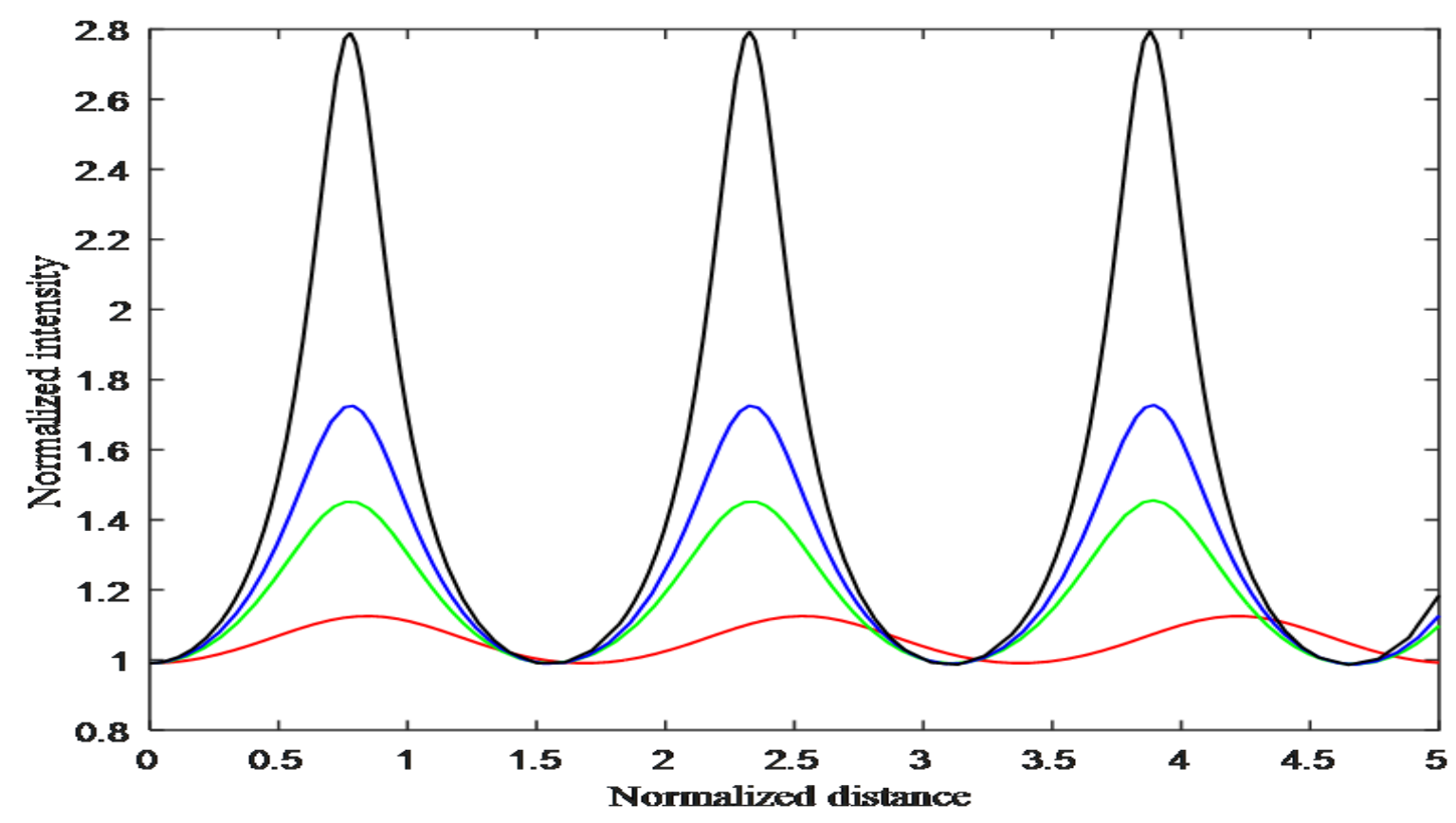

Figure 3. Variation of the normalized intensity of cosh-Gaussian laser beam in the plasma with the normalized distance of propagation $(\xi)$ for different values of $b$ in non-paraxial region, when relativistic and ponderomotive nonlinearities are operative. Keeping $a=1.4$ and $\omega_{p 0}=0.06 \omega_{0}$. Red, Green, Blue and black curves for $b=0,0.4,0.6$ and 0.8 respectively.

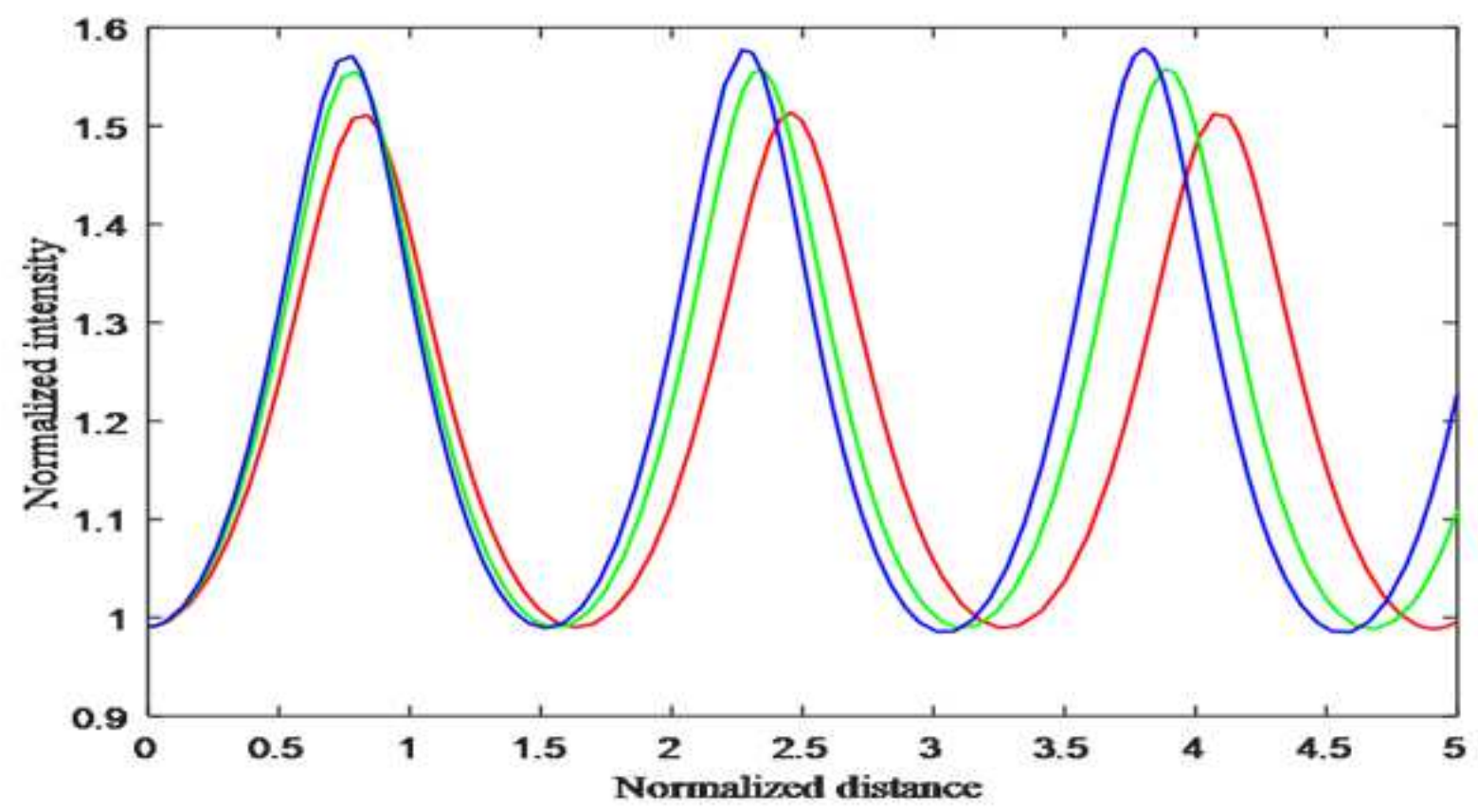

Figure 4. Variation of the normalized intensity of cosh-Gaussian laser beam in the plasma with the normalized distance of propagation ( $\xi$ )for different values of $a$ in non-paraxial region, when relativistic and ponderomotive nonlinearities are operative. Keeping $b=0.6$ and $\omega_{p 0}=0.06 \omega_{0}$. Red, Green, and Blue curves for $a=1,1.4$, and 2 respectively. 


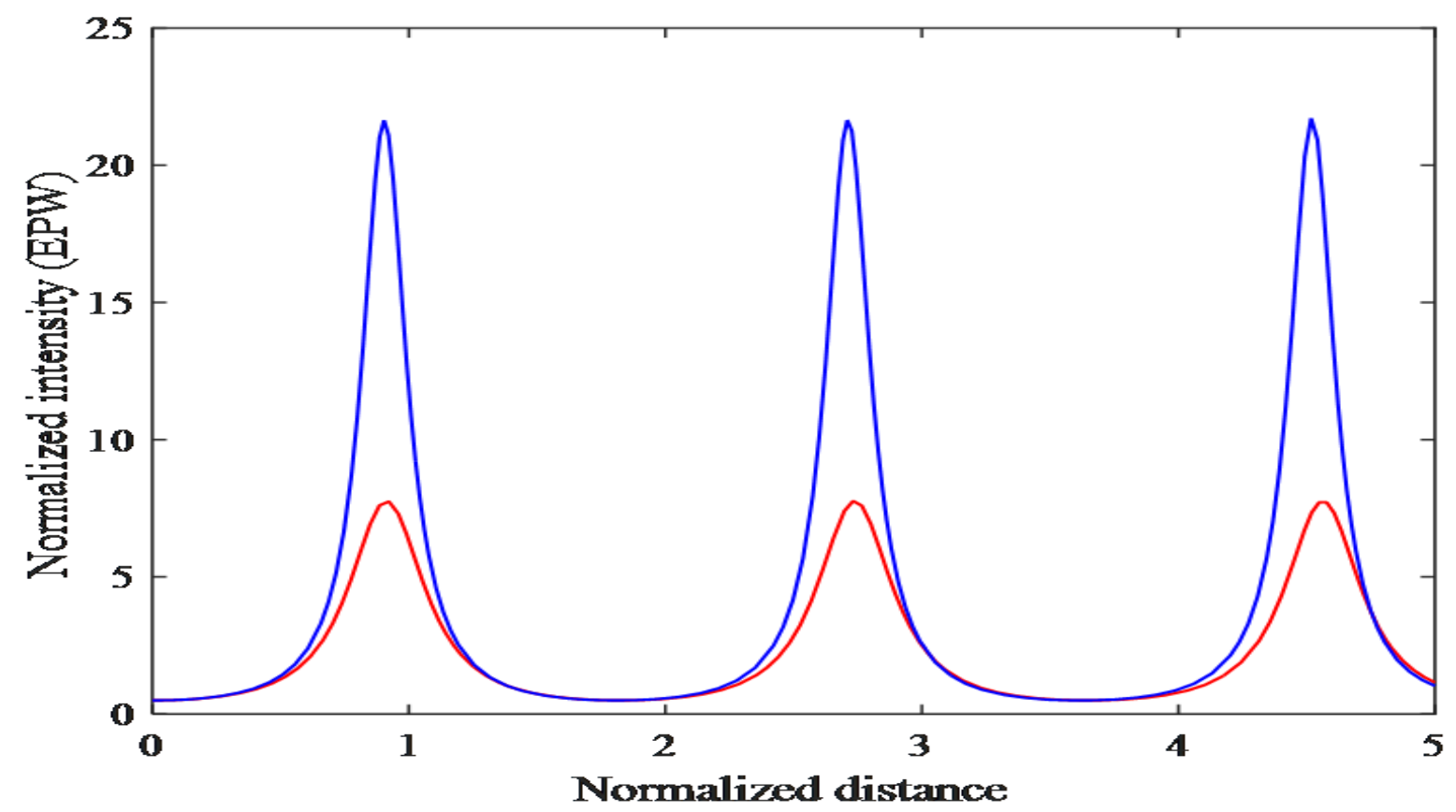

Figure 5. Variation of normalized intensity of EPW with normalized propagation distance $(\xi)$. Keeping $a=1.4, b=0.6$, and $\omega_{p 0}=0.06 \omega_{0}$, when relativistic and ponderomotive nonlinearities are operative. [Red curve for paraxial region and blue curve for non-paraxial region].

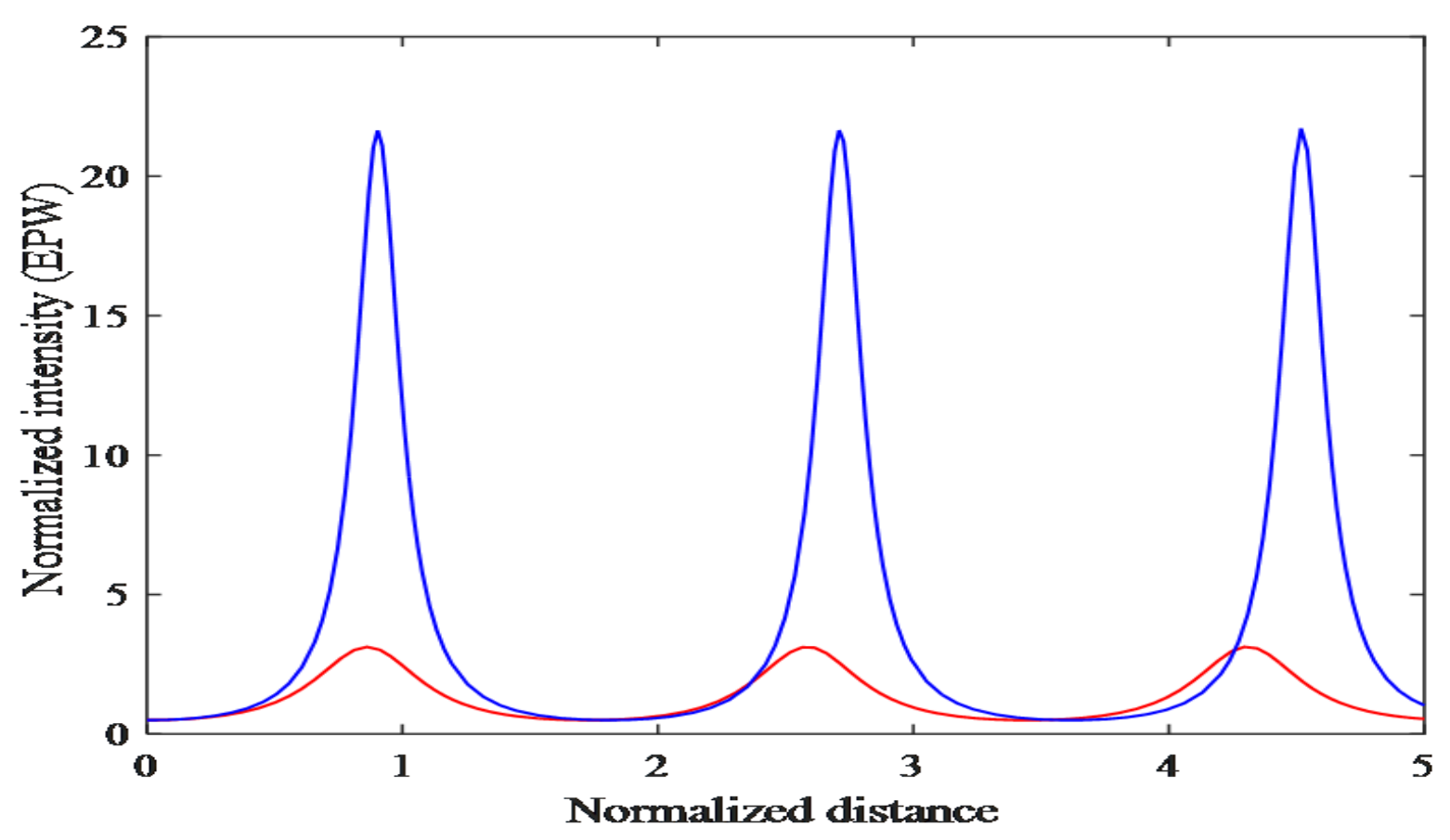

Figure 6. Variation of normalized intensity of EPW with normalized propagation distance $(\xi)$. Keeping $a=1.4, b=0.6$, and $\omega_{p 0}=0.06 \omega_{0}$, when only relativistic and both relativistic and ponderomotive nonlinearities are operative. Red curve for only relativistic and blue curve for relativistic ponderomotive nonlinearities. 


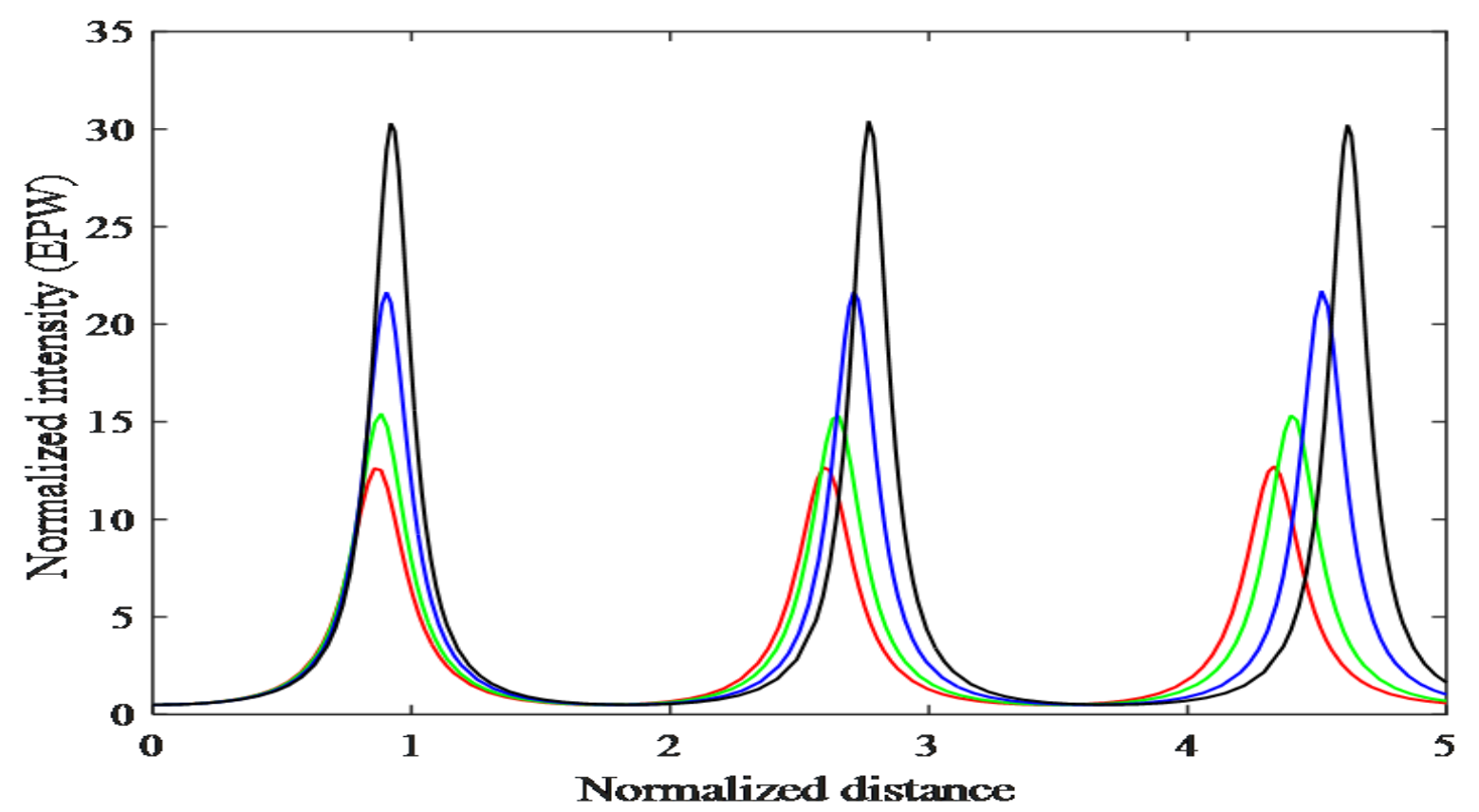

Figure 7. Variation of the normalized intensity of EPW with the normalized distance of propagation $(\xi)$ for different values of $b$ in non-paraxial region, when relativistic and ponderomotive nonlinearities are operative. Keeping $a=1.4$ and $\omega_{p 0}=0.06 \omega_{0}$. Red, Green, Blue and black curves for $b=0,0.4,0.6$ and 0.8 respectively.

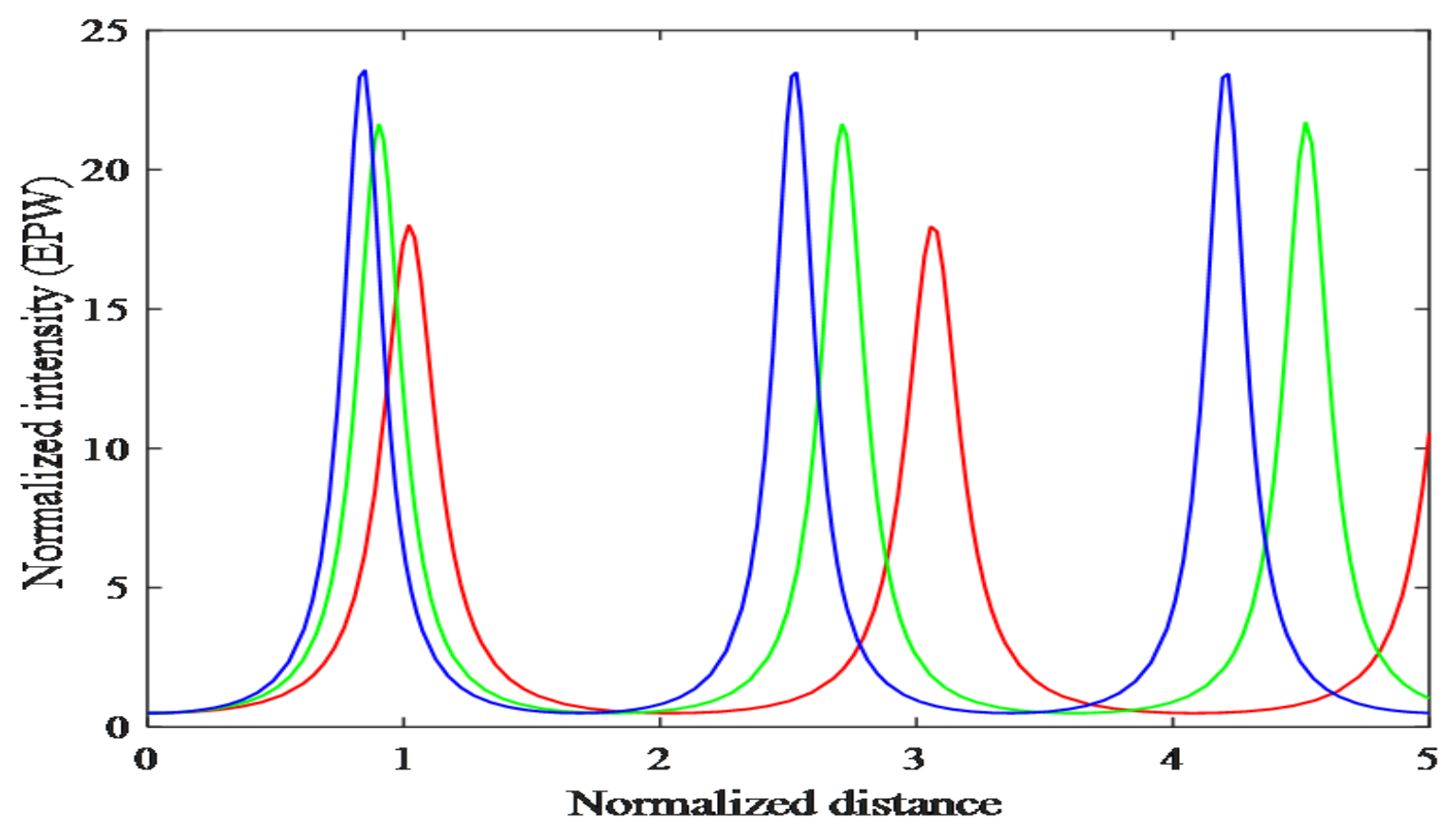

Figure 8. Variation of normalized intensity of EPW with the normalized distance of propagation $(\xi)$ for different values of $a$ in non-paraxial region, when relativistic and ponderomotive nonlinearities are operative. Keeping $b=0.6$ and $\omega_{p 0}=0.06 \omega_{0}$. Red, Green, and Blue curves for $a=1,1.4$, and 2 respectively. 


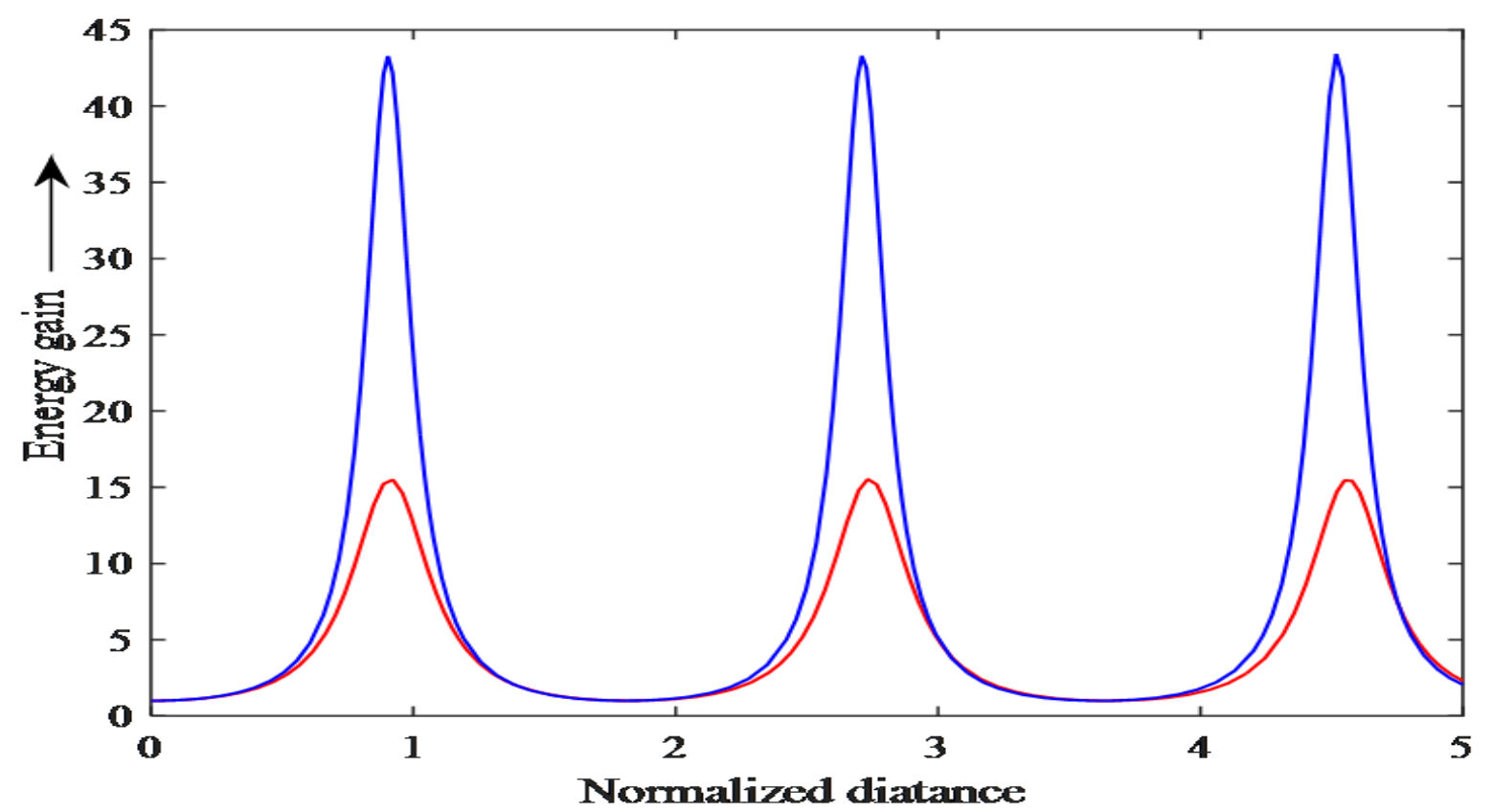

Figure 9. Variation of energy gain with normalized propagation distance $(\xi)$. Keeping $a=1.4$, $b=0.6$, and $\omega_{p 0}=0.06 \omega_{0}$, when relativistic and ponderomotive nonlinearities are operative. Red curve for paraxial and blue curve for non-paraxial region.

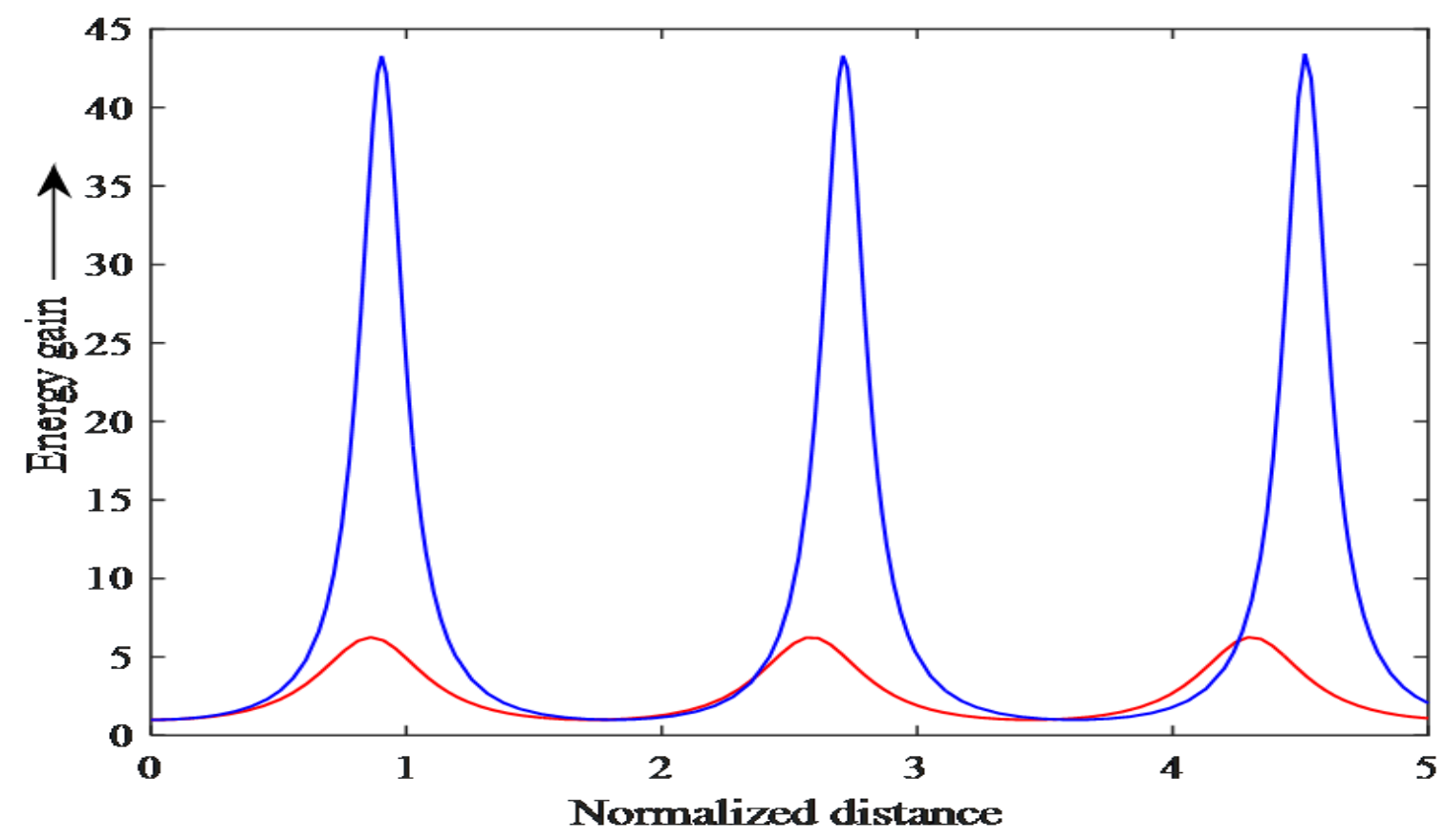

Figure 10. Variation of energy gain with normalized propagation distance $(\xi)$. Keeping $a=$ $1.4, b=0.6$, and $\omega_{p 0}=0.06 \omega_{0}$, when only relativistic and both relativistic and ponderomotive nonlinearities are operative. Red curve for only relativistic and blue curve for relativistic ponderomotive nonlinearities. 


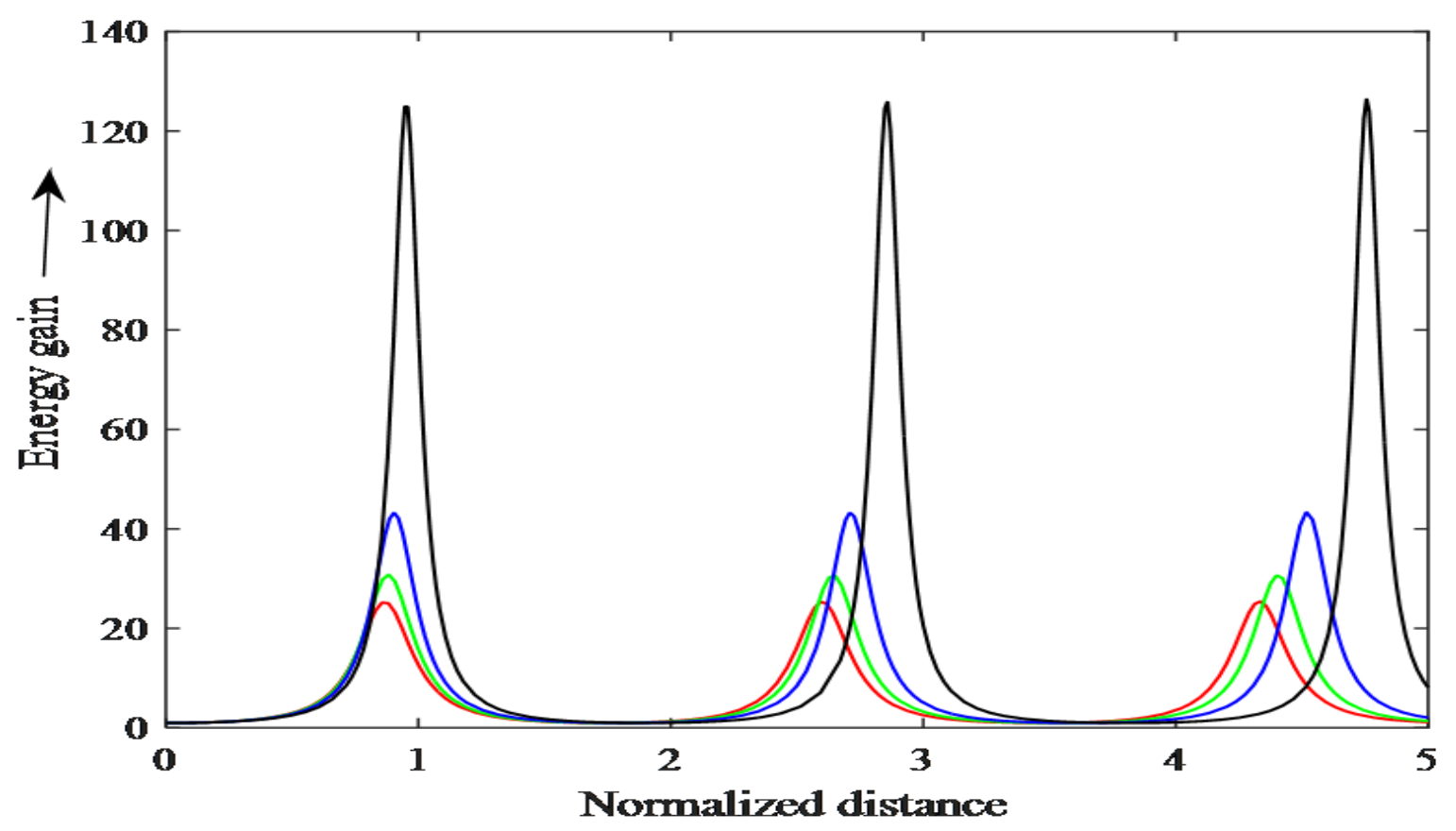

Figure 11. Variation of the energy gain with the normalized distance of propagation $(\xi)$ for different values of $b$ in non-paraxial region, when relativistic and ponderomotive nonlinearities are operative. Keeping $a=1.4$ and $\omega_{p 0}=0.06 \omega_{0}$. Red, Green, Blue and black curves for $b=0$, $0.4,0.6$ and 0.8 respectively.

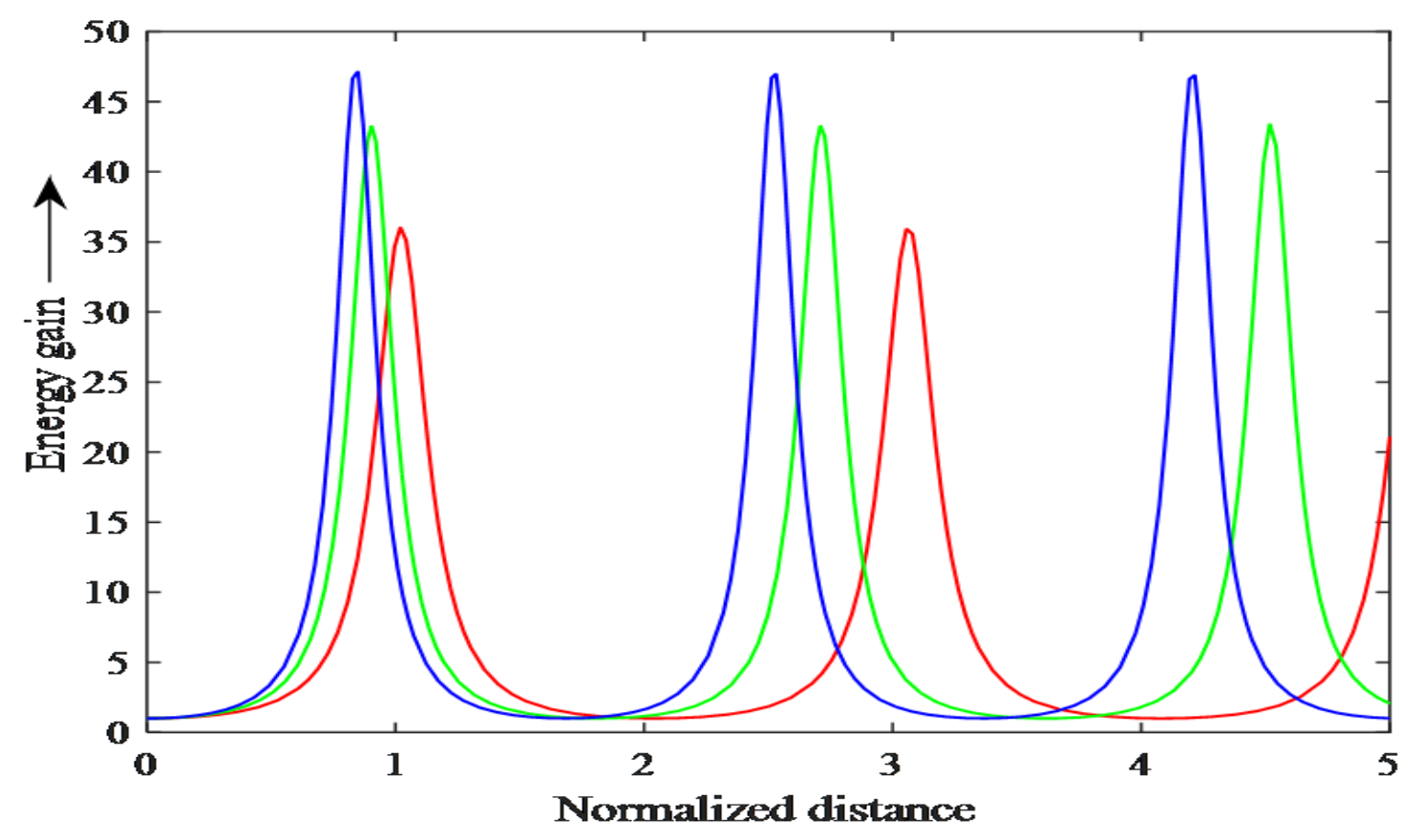

Figure 12. Variation of energy gain with the normalized distance of propagation $(\xi)$ for different values of $a$ in non-paraxial region, when relativistic and ponderomotive nonlinearities are operative. Keeping $b=0.6$ and $\omega_{p 0}=0.06 \omega_{0}$. Red, Green, and Blue curves for $a=1,1.4$, and 2 respectively. 